Martin Hinton

University of Łódź

ORCID 0000-0003-0374-8834

Marcin Koszowy

University of Białystok \& Polish Academy of Sciences

ORCID 0000-0001-5553-7428

\title{
INTRODUCTION TO THE ISSUE: THE PHILOSOPHY OF ARGUMENTATION
}

The practice of argumentation is ubiquitous, both in everyday life and, under more rigorous control, one hopes, in the sphere of academic research and debate. It is natural, therefore, that, since its influence spreads in many directions, it can itself be investigated from many angles and approached by many routes. Arguments can be considered from the perspective of discourse studies, dialectic and pragmatics; their language can be examined by linguists and rhetoricians; their motives and effects by psychology, political science and even the ever-creative practitioners of marketing and advertising. All of these approaches are of value, and it is the multi-faceted nature of the modern field of Argumentation which makes it so relevant, so dynamic, and so fascinating.

It is within philosophy, however, that the study of argumentation as reasoning began and where it continues today; influenced and improved, no doubt, by contact with those assessing it from other vantage points; but focussed still on the structure of arguments, and on their relation to logic and to the wider philosophical issues of truth, knowledge and the nature of the human mind.

This issue brings together the work of a number of scholars; some of them international leaders in the field, others young researchers with new ideas; but all taking a philosophical approach to the study of argument. The content of their work is reviewed briefly below, but it should first be noted how this issue is also a testament to the strength of the Argumentation scene in Poland. This is not so much reflected in the nationality or affiliation of the contributors - only the editors work at Polish universities - but rather by the reach of events, publications and organisations originating in the country. Several of the papers in this volume were originally presented 
at events at the University of Łódź: at the Workshop in Informal Logic and Linguistics (WILL), which has just seen its second edition, and at the Argumentation Sessions of PhiLang 2017, which will again be held at the conference in 2019, under the name PhilArg.

These events, however, represent only a small part of the wider activity of researchers at Polish institutions and Polish researchers around the world. Others cycles of conferences include Poznań Reasoning Week, and the meetings of ArgDiaP, which this year became an officially recognised scientific organisation. ArgDiaP events include various workshops, as well as the main conference, in its $16^{\text {th }}$ edition this year which is reported on in this issue as a part of the Warsaw Argumentation Week (WAW 2018). These events and the contacts and publications they spawn have made Poland the hub of argumentation research in the Central and Eastern European regions. The globally interconnected nature of Polish efforts in this field is reflected by the diversity of affiliations and backgrounds of both the contributors to this issue, and their reviewers, who are listed below.

The most obvious sign of Polish influence comes in the extended essay, which concludes the volume, by Ralph Johnson and Marcin Koszowy, Logical Culture as a Common Ground for the Lvov-Warsaw School and the Informal Logic Initiative. In this paper, the authors show how the Polish philosophical tradition of logic is related to and reflected in the more recent North American initiative of Informal Logic. What they share, and what is still a vital part of the Polish scene today, is a belief in education in logical thinking; a belief that such an education can allow those who receive it to better express and understand arguments employed in all areas of life; a belief, fundamentally, in what they call a 'logical culture'.

Several of the papers in this issue deal with questions of the most fundamental nature in the study of argumentation, and it is with those that we begin, before moving on to papers in which theoretical approaches are applied to individual cases. The first such work, then, concerns the ethics of argumentative discussion. In Norms in Deliberation: The Role of the Principles of Justice and Universalization in Practical Discourses on the Justice of Norms, Cristina Corredor assesses the role of John Rawls's principle of justice and Jürgen Habermas's principle of universalization in deliberations on justice.

Erich Rast has provided a contribution detailing concepts from formal argumentation concerned with the assessment of argument strength. In Towards a Model of Argument Strength for Bipolar Argumentation Graphs, he sets out conditions for the combination of the strengths of sub-arguments into an overall complex argument strength. 
A third fundamental issue addressed within these pages is the classification and categorisation of types of argument. Jean Wagemans has created a great deal of interest with his innovative construction of a Periodic Table of Arguments. In Analogy, Similarity, and the Periodic Table of Arguments, he explains the theoretical background to the table and shows how arguments from analogy fit into that overall framework.

Martin Hinton tackles the topic of deep disagreement, first raised in Fogelin's famous paper. He suggests in Overcoming Disagreement through Ordering: Building an Epistemic Hierarchy that apparently deep disagreement may be only the result of a difference in prioritisation of certain beliefs and that a discussion of hierarchies may provide a way to move forward discussions which have reached an impasse.

The first paper to look in detail at a particular scheme of argument is from renowned scholar Douglas Walton. In this work, Witness Impeachment in Cross-Examination Using Ad Hominem Argumentation, Walton shows how the technique of constructing conflict diagrams can be used to draw inferences concerning the reliability of witness statements in court room cross-examinations.

Miklós Könczöl addresses another well-known, but not well-loved, form of argument in Ad misericordiam revisited. Here, he gives an overview of earlier work on such appeals and suggests a more traditional, logic-based, narrowing of the definition to include only those examples which are fallacious under the term.

The next two papers look at argumentation in science. In $A$ new Argument-Scheme for Causal Explanations by Analogy? - The Case of Galileo's Explanation of the Tides, Alexander Kremling looks at how Galileo explained the existence of tides and argues that it represents a form of argument from analogy useful in science. Carlo Martini, in his paper, Ad Hominem Arguments, Rhetoric, and Science Communication, examines the use of effective persuasive strategies to complement evidence-based approaches in the public communication of science, specifically within the context of the debate over vaccine use.

Finally, David Botting examines the use of discounting expressions in the conclusions of arguments. His paper, "Even Though": On the Different Functions of Discounting Expressions in Pro and Con Arguments, argues that the prevalence of such expressions is evidence for the existence of conductive arguments.

The works presented above certainly illustrate the wide range of research being done within the philosophical approach to argumentation and, we hope, make a contribution to the furtherment of knowledge and under- 
standing of these important topics. They have also required a wide range of reviewers, who have provided an invaluable service in the selection and improvement of these papers. The editors would like to express their sincere gratitude to the following scholars who read and reviewed the contributions, making the production of this volume possible: Linda Carozza (York University), Ian Dove (University of Nevada), David Godden (Michigan State University), Jean Goodwin (NC State University), Henrike Jansen (University of Leiden), Marcin Lewiński (New University of Lisbon), Steve Oswald (University of Fribourg), Steven Patterson (Marygrove College), Juho Ritola (University of Turku), Piotr Stalmaszczyk (University of Łódź), Douglas Walton (University of Windsor), and Frank Zenker (University of Lund). 